\title{
GLADE \\ Global Liquid Argon Detector Experiment \\ A Letter of Intent to FNAL
}

The recent measurements of the $\theta_{13}$ mixing angle, which controls the observable size of any $\mathrm{CP}$ violation effects, open a window of opportunity to take advantage of the world's most powerful existing neutrino beam together with recent successes in development of the ultimate detector technology for the detection of electron neutrinos : a liquid argon ( LAr) time projection chamber. During this proposed project a 5kt LAr detector (GLADE) will be developed by European groups to be put in a cryostat in the NuMI neutrino beam at Fermi National Accelerator Laboratory in the US and will start taking data in 3-5 years time to address the neutrino mass ordering.

The successful fruition of this project, along with nominal exposure at $\mathrm{NO} \nu \mathrm{A}$ and $\mathrm{T} 2 \mathrm{~K}$, together with information from double beta decay experiments could ascertain that neutrinos are Dirac particles in the next decade.

\section{Overview}

The discovery of neutrino mass was one of the major scientific advances of the last two decades. One of the most successful theories known to mankind is the Standard Model (SM) of Glashow, Salam and Weinberg, which accounts for the interactions of the constituents of matter and three of the four known forces of Nature. Neutrinos belong to one of the two types of fermionic constituents: they are called leptons and they carry no charge. There are three flavours of neutrino $\left(\nu_{e}, \nu_{\mu}\right.$ and $\left.\nu_{\tau}\right)$ and they have half-integer spin which can be aligned either in the direction of their momentum (right-handed) or against it (left-handed). In the SM, the Weak Interaction is the only force which neutrinos can 'see' and it only 'sees' left-handed neutrinos. The SM therefore assigned neutrinos exactly zero mass in order to avoid there being sterile particles with no interactions with any of the three forces. Mass connects left-handed and righthanded spin states and therefore produces right-handed neutrinos which do not have a gauge interaction and can be thought of as sterile. Massive neutrinos are the first evidence of particle physics not described by the SM.

Experiments have now confirmed that neutrinos indeed have mass, that they oscillate from one flavour to another in flight and that they seem to conform to a simple $3 \times 3$ mixing matrix put forward by Pontecorvo, Maki, Nakagawa and Sakata (PMNS) most of whose parameters have already been measured to an accuracy of about $10 \%$ over the past 10 years. This $3 x 3$ matrix, which connects the Weak Interaction states with the mass states which actually propagate, has provided a framework in which to present the ever more precise measurements being made. There are now new questions which have arisen:

1. what is the ordering of the masses of the neutrinos?

2. are neutrinos responsible for the matter-antimatter asymmetry in the Universe?

3. what is the fundamental nature of the neutrino (Dirac or Majorana)?

On the way to answering these questions, there are two notable ground breaking measurements which will need to be made, that of looking for differences between electron neutrinos $\left(\nu_{e}\right)$ and antineutrinos $\left(\overline{\nu_{e}}\right)$ to identify the mass ordering of the three neutrinos; then to disentangle this feature from the effects of $\mathrm{CP}$ violation. 
The mass ordering is sensitive to the electron density that the $\nu_{\mathrm{e}}$ traverse which is a known SM process and affects $\nu_{\mathrm{e}}$ and $\overline{\nu_{\mathrm{e}}}$ differently. Knowing the mass ordering alone could lead to a clear identification of the fundamental nature of the neutrino if the result is that $m_{3}$ is lighter than $\mathrm{m}_{1}$ and $\mathrm{m}_{2}$ and there is no observation of neutrino-less double beta decay (DBD) in the next generation DBD experiments. In this case, neutrinos would be proved to be Dirac particles.

$\mathrm{CP}$ violation on the other hand is by definition the difference between interactions of particles and anti-particles, and remains to be discovered in the neutrino sector. The accurate detection of the $\nu_{\mathrm{e}}$ and $\overline{\nu_{\mathrm{e}}}$ events is paramount in these two endeavors. Once the mass hierarchy is determined, the $\mathrm{CP}$ violation angle can be measured with the combination of GLADE, NO $\nu$ Aand T2K with some accuracy depending on its value.

The overarching objective of this proposal is to uncover the next level of detail in our understanding of the neutrino. Particle physics has become global. The scale of the experiments is so large that they can no longer be mounted by any one local geographical region. The goal of the proposal is to enhance the European involvement and leadership in a new global project.

\section{GLADE in the NuMI beam}

Presently, the absolute masses of the neutrinos are not known, only mass squared differences which come from the oscillation experiments, and while we know that there are two neutrinos with a small difference between their masses $\left(\mathrm{m}_{1}\right.$ and $\left.\mathrm{m}_{2}\right)$ and one with a large difference from those other two $\left(m_{3}\right)$, we do not know whether $m_{3}$ is lighter (Inverted Hierarchy) or heavier (Normal Heirarchy) than the other two. Furthermore, the value of the lightest neutrino mass is not known. Results from MINOS[3], T2K[4], Chooz, DoubleChooz[5] and most recently Daya Bay[6], as shown in Figure 1 (top), point to a value of $\theta_{13} \sim 8.8^{\circ}$, which, while small, is of a magnitude which has nevertheless opened up a window of opportunity for the existing long baseline neutrino experiments which this proposal seeks to exploit. New reactor experiments, further running of the $\mathrm{T} 2 \mathrm{~K}$ experiment and the first data from $\mathrm{NO} \nu \mathrm{A}$ should pin down the $\theta_{13}$ angle with very high accuracy in the coming two years. It should then be possible, with the extra reach extended by this proposal, to get a very good indication of the mass hierarchy.

The more difficult goal is to identify the size of $\mathrm{CP}$ violation in the neutrino sector. The small size of the $\theta_{13}$ mixing angle which controls the observable size of the $\mathrm{CP}$ violation effects in $\nu_{\mathrm{e}}$ and $\overline{\nu_{\mathrm{e}}}$ interactions, requires detector technologies well suited to seeing very small numbers of dense electron core events above large and quite similar looking background events. If CP violation can be observed, it could give us an example of a way in which the matter-anti-matter asymmetry could have been produced in the early universe.

The ultimate detector technology for the detection of electron neutrinos uses Time Projection Chamber (TPC) technology in a liquid argon (LAr) volume. Gas filled TPC's have been used successfully for many years : they provide very accurate 3 -D tracking information, where $\mathrm{x}$ and $\mathrm{y}$ are provided by the positions of the components which eventually collect the charge while the $\mathrm{z}$ position is given by the time that the signals arrive compared to the start of the event (T0). In the LAr case, liquid argon scintillates when a charged track traverses it, and this scintillation light can be detected and used to signal the start of the event. The electrons produced in the ionization made by charged particles traversing the volume of the LAr drift under the influence of a constant electric field to the end detectors which can be wires, interpolating pads or gas electron multipliers (GEMs), where the time and charge is recorded. This constitutes a great advance for neutrino detection because the liquid is very heavy, and so a large mass of detector can be presented to the very weakly interacting neutrinos, which at the same time is combined with excellent 3D tracking information. This has recently been demonstrated by the $600 \mathrm{~T}$ ICARUS experiment in the CNGS beam. Smaller detectors have been developed and run at FNAL and elsewhere. 

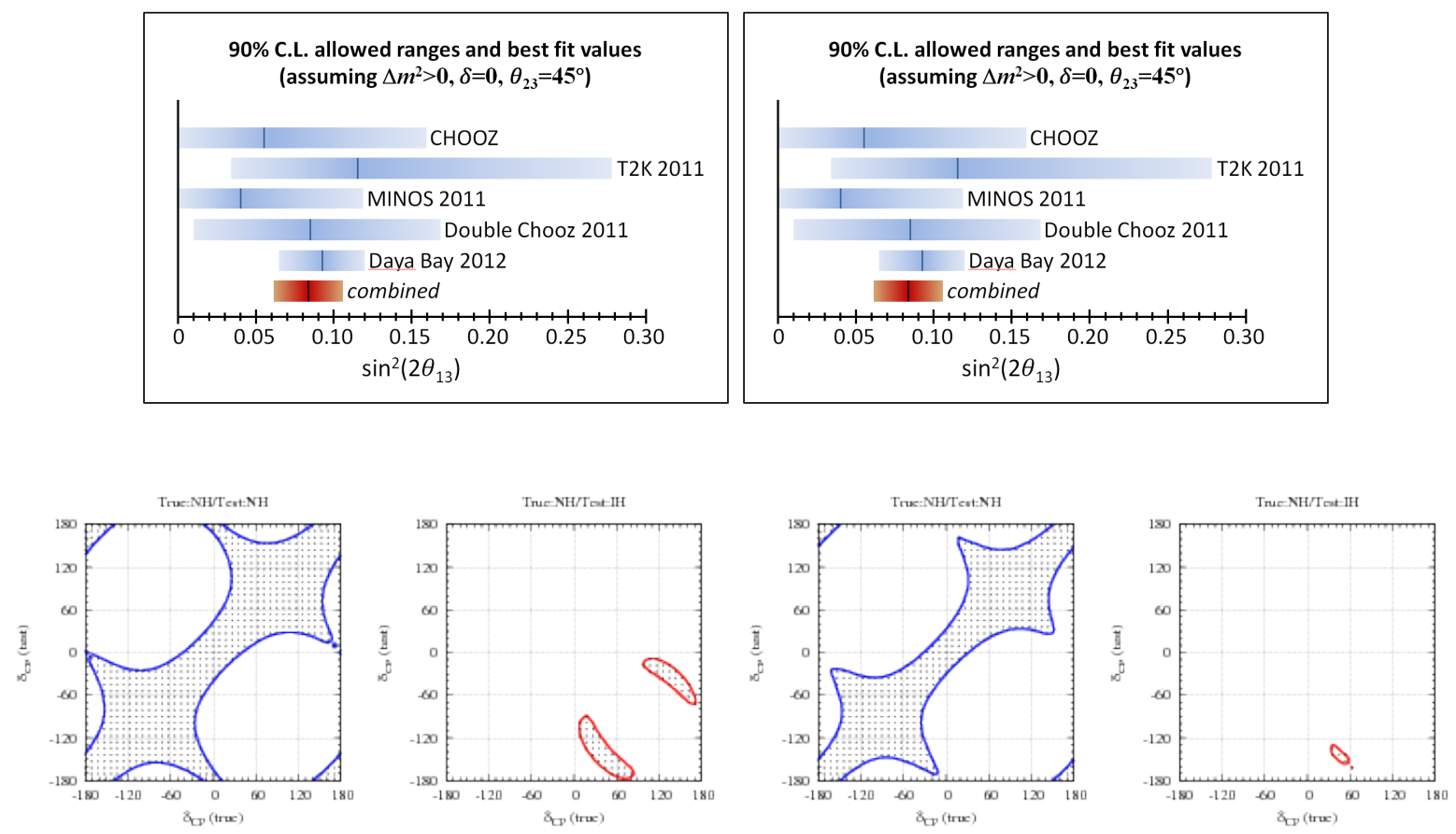

Figure 1: Top: The present knowledge of the parameter $\theta_{13}$ (Courtesy Ryan Patterson). Bottom: the left two plots show the $90 \%$ remaining allowed $\delta_{C P}$ space after the nominal running of $\mathrm{NO} \nu \mathrm{A}(5+5)$ and $\mathrm{T} 2 \mathrm{~K}(6+0)$. On the left is the allowed $\delta_{C P}$ region (shaded) using the correct (in this case NH) hypothesis, while on the right is the test using the incorrect $(\mathrm{IH})$ hypothesis. The two right plots show the expected reach of GLADE $(2+5$ : neutrino+anti-neutrino) in combination with $\mathrm{NO} \nu \mathrm{A}(5+5)$ and $\mathrm{T} 2 \mathrm{~K}(6+0)[2]$. Including GLADE, the incorrect mass hierarchy hypothesis can be ruled out at somewhere close to $90 \%$. The exact C.L. depends on the relative power of GLADE compared to $\mathrm{NO} \nu \mathrm{A}$, the total overall exposure and the actual value of $\theta_{13}$.

It is time to foster a new international collaboration with significant European involvement to build a 5kt liquid argon detector, GLADE (Global Liquid Argon Detector Experiment), in the already existing, and to be upgraded, NuMI beam at FNAL, the present and near-future most powerful neutrino beam in the world. It will be located off-axis on the surface at the Ash River site, $810 \mathrm{~km}$ from the neutrino source, in the same hall as the $\mathrm{NO} \nu \mathrm{A}$ detector presently in construction. It would start taking data in 3-4 years time.

The physics reach is compelling. Figure 1 (bottom) shows the allowed region in $\delta_{C P}$ remaining after 7 years of GLADE running ( 2 with neutrinos and 5 with anti-neutrinos), when combined with the planned $\mathrm{NO} \nu \mathrm{A}(5+5)$ and $\mathrm{T} 2 \mathrm{~K}$ runs $(6+0)$ and the reactor experiments. While with the nominal running of just $\mathrm{NO} \nu \mathrm{A}$ and $\mathrm{T} 2 \mathrm{~K}$ there is a substantial region of allowed $\delta_{C P}$ space in the wrong hierarchy plot (left), almost the entire incorrect-hierarchy solution would be ruled out with the addition of GLADE information at 90\% confidence level (right). The reactor experiments and $\mathrm{T} 2 \mathrm{~K}$ provide information which is not dependent on the mass hierarchy and so they are needed to disentangle the matter effects from $\mathrm{CP}$ violation. The reactor experiments have no $\mathrm{CP}$ violation while the $\mathrm{T} 2 \mathrm{~K}$ experiment has very little matter effect. At worst, if $\theta_{13}$ ends up being much lower than 0.08 , the results from this proposal will constitute the best limits in the world and certainly will provide a strong indication of which hierarchy is favoured. At best, if the performance of the GLADE detector turns out to be better than $3 \mathrm{x}$ that of $\mathrm{NO} \nu \mathrm{A}$ or if the NuMI beam runs for longer than 10 years, this exclusion could be higher than 90\%C.L.

Figure 2 left shows a potential running strategy. If it is assumed that T2K only runs for the 
nominal 6 years in neutrino mode at design intensity of $0.77 \mathrm{~kW}$, then the necessary complementary information can be delivered by the $\mathrm{NO} \nu \mathrm{A}+\mathrm{GLADE}$ experiments. The left plot shows the confidence level at which the incorrect hierarchy can be ruled out as a function of collected $\mathrm{NO} \nu \mathrm{A}$ neutrino and anti-neutrino years. ( $1 \mathrm{NO} \nu \mathrm{A}$ year is expected to be equivalent to $1 \mathrm{GLADE}$ year). In the nominal $\mathrm{T} 2 \mathrm{~K}$ scenario, the $\mathrm{NO} \nu \mathrm{A} / \mathrm{GLADE}$ combination can provide enough exposure to achieve a 90-95\%C.L. exclusion of the wrong hierarchy inside the planned NuMI running time.
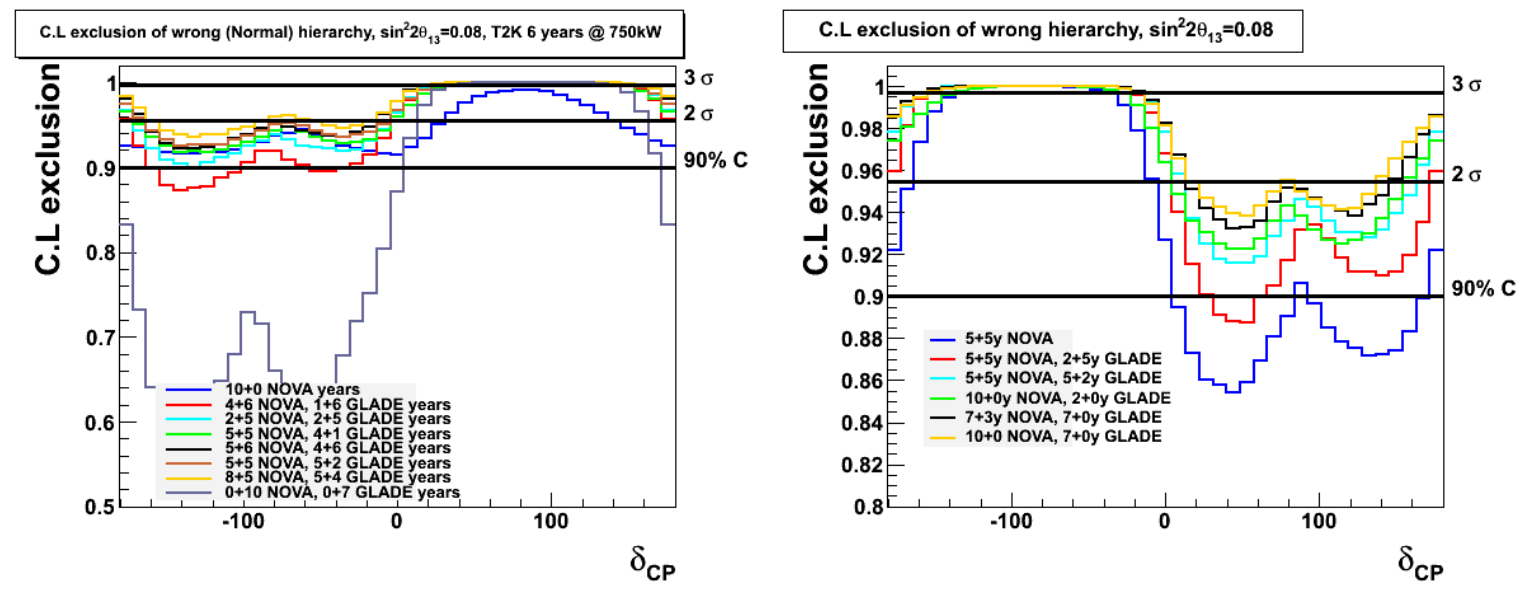

Figure 2: The C.L. level exclusion of the wrong mass hierarchy by the NO $\nu$ A/GLADE/T2K combination given $6+0$ (neutrino+anti-neutrino) years of T2K running as a function of GLADE and NO $\nu$ Arunning. Left Inverted Hiearchy, Right, Normal Hierarchy. This assumes a value of $\sin ^{2} 2 \theta_{13}$ of 0.08 and that one GLADE year $\equiv$ one $\mathrm{NO} \nu \mathrm{A}$ year.

The detector design will likely follow that developed by the ETH group using Gas Electron Multiplier devices[7]. The major development would take place at CERN where a small cryostat will be installed for testing, thereby giving a number of other European collaborators access to the hardware development and commissioning before the finished inner detectors are shipped to the US for exploitation.

The proposal has been discussed with the CERN management which is now considering increasing support for studies of next generation neutrino detectors, in the context of the rolling CERN R\&D program, with the goal of encouraging a truly global effort in this area. Adequate space, a test cryostat and some technical effort is being formally requested as a contribution to the project.

\section{Request for Resources}

This letter of intent requests an indication from FNAL Directorate of the appetite of the laboratory to mount such an experiment. Specifically, in order to be successful, the cryostat infrastructure, purification, and Liquid Argon would have to be provided by FNAL. A clear indication of this intent would enable the coalescence of the European groups towards mounting such an effort at FNAL. This idea is new (largely owing to the very recent results on $\theta_{13}$ ) but has been met with significant enthusiasm from a number of notable European specialists. Funds from the countries' agencies would be requested in the coming one to two years as the collaboration is formed.

This project will act as a prototype for the next generation of long baseline experiments. Presently there are plans at FNAL for the next generation LBNE project which has a $1300 \mathrm{~km}$ baseline and will deliver broad coverage of the mass ordering and CP violation. There are also 
discussions about an even longer baseline experiment at CERN. The European/US collaboration fostered during this project will be well positioned to take advantage of whichever project is realized.

\section{World Knowledge}

The main characteristic of neutrinos, that they are weakly interacting, makes studying them a challenge. Their interactions are very rare and experiments are already effectively combining their neutrino events in some way in order to extract the maximum information about these elusive particles. This will become even more necessary in the future. One rather different set of experiments from those described above, which actually look for events which contain NO neutrinos, will be reaching new sensitivities for the neutrino-less double beta decay process in the coming decade. Such decays are allowed if the neutrino is its own anti-particle (Majorana) but forbidden if the neutrino and its anti-particle are distinct (Dirac), like all the other fermions. The neutrino is the only known fundamental fermion which could have this characteristic, because it is electrically neutral. Interestingly, the combination of the double beta decay results and those of the long baseline experiments could make a breakthrough in understanding the fundamental particle type of the neutrino in the next decade. If the long baseline experiments are able to ascertain that the mass hierarchy is actually inverted, and the DBD experiments do not see any neutrino-less double beta decay events, one will be able to conclude that neutrinos are Dirac

particles. While this requires a number of of results to line up, its knowledge reward would be immense if successful.

\section{References}

[1] Pedro Machado, Private Communication, updated from arXiv:1111.3330v1 [hep-ph] 14 Nov 2011

[2] Sushant Raut, Private Communication , based on arXiv:1201.6485v1 [hep-ph] 31 Jan 2012

[3] P. Adamson et al. (MINOS Collaboration), Phys. Rev. Lett. 107,181802 (2011).

[4] K. Abe et al. (T2K Collaboration), Phys. Rev. Lett. 107, 041801(2011).

[5] arXiv:1112.6353v2 [hep-ex] 27 Jan 2012

[6] (Daya Bay collaboration) http://arxiv.org/abs/1203.1669v1

[7] A. Badertscher et al. arXiv:1012.0483v1 